\title{
Pheochromocytoma Multisystem Crises Treated with Emergency Surgery: A Case Report
}

\section{Elisabete Coelho*, Laura Costa, Fátima Assunção and Luís Lencastre}

Unidade de Cuidados Intensivos Polivalente, Hospital de Braga, Braga, Portugal

*Corresponding Author: Elisabete Coelho, Unidade de Cuidados Intensivos

Polivalente, Hospital de Braga, Braga, Portugal.
Received: March 25, 2021

Published: May 19, 2021

(C) All rights are reserved by Elisabete

Coelho., et al.

\begin{abstract}
The authors describe a case of a 50-year-old woman admitted in the emergency room with a shock with multiorgan disfunction. An adrenal mass was identified in the initial approach. She was admitted in the intensive care unit and had a progressive worsening to multiorgan failure. The diagnosis of a pheochromocytoma multisystem crisis was done and she was treated successfully with emergency surgery. After surgery, however, an irreversible ischemia of her right hand and both limbs persisted leading to several amputations. After plastic surgery intervention, adaptation to prosthetics and functional rehabilitation, the patient was able to return to her life.
\end{abstract}

Keywords: Pheochromocytoma; Sympathomimetic Effects; Multiple Organ Failure

\section{Background}

Pheochromocytomas are rare catecholamine-producing tumours originating from chromaffin cells in the adrenal medulla or sympathetic paraganglia. It is most common in the fourth to fifth decade and equally frequent in both men and women [1]. The classic triad (paroxysmal headache, sweating, tachycardia) is present in a minority of the cases [1]. These tumours may display numerous clinical manifestations because of hemodynamic and metabolic effects of high circulating catecholamine levels [2]. These symptoms are often severe, and some patients may present with a pheochromocytoma multisystem crisis (PMC) [3].

PMC is a fatal condition characterized by multiorgan failure, severe blood pressure variability, high fever, and encephalopathy. Symptoms may vary greatly; abdominal pain, nausea and dyspnoea are common, while anemia, back pain, night sweats and acidosis have also been reported [3].

We report a case of PMC treated successfully with emergency surgery, complicated with irreversible ischemia of the limbs.

\section{Case Report}

We present a case of a 50-year old caucasian woman, with no past medical history, except for a light recurrent headache. She did not take any medication and she had no known allergies.

She was brought to the hospital by her relatives who had found her lying on the floor poorly reactive and hypothermic. She had been complaining of persistent abdominal pain, nausea and vomiting for 2 days.

At admission, in the emergency room, she was tachypnoeic, with low peripheric oxygen saturation, and her lung sounds were globally diminished. She had sinus tachycardia with normal blood pressure. Her face was plethoric, but she showed a delayed capillary refill with exuberant generalized mottling. She was agitated, uncooperative and hypothermic.

The gasometry showed a type 1 respiratory insufficiency, a metabolic acidemia with elevated anion gap and elevated lactate. Laboratory results revealed an increased creatinine, leucocytosis, and a slight elevation of C-reactive protein (Table 1). The echocardiogram presented a severe depression of the left ventricular systolic function, with marked apical hypokinesia, but with preserved contractility in the basal segments. The computed tomography (CT) scan revealed an heterogenous nodular lesion in the left adrenal gland (65 x $45 \mathrm{~mm}$ ) (Figure 1). 


\begin{tabular}{|c|c|c|c|c|}
\hline & Admission & Before Surgery & Discharge ICU & Reference values \\
\hline \multicolumn{5}{|c|}{ Gasometry } \\
\hline $\mathrm{pH}$ & 6,974 & 7,345 & 7,44 & $7,35-7,45$ \\
\hline $\mathrm{pO}^{2}$ & 76,6 & 121 & 97 & $70-100 \mathrm{mmHg}$ \\
\hline $\mathrm{pCO}^{2}$ & 31,3 & 37,9 & 30,5 & $35-45 \mathrm{mmHg}$ \\
\hline $\mathrm{HCO}_{3}^{-}$ & 7,1 & 20,3 & 20,4 & $21-26 \mathrm{mmol} / \mathrm{L}$ \\
\hline Lactate & 7,95 & 5,76 & 1,46 & $0,5-2,0 \mathrm{mmol} / \mathrm{L}$ \\
\hline \multicolumn{5}{|c|}{ Hematology/Biochemistry } \\
\hline Hemoglobin & 16,1 & 7,5 & 7,7 & $11,9-16,6 \mathrm{~g} / \mathrm{dL}$ \\
\hline Hematocrit & 49,2 & 22,8 & 24,1 & $36,6-45 \%$ \\
\hline White blood count & 20,9 & 18,29 & 12,68 & $4.0-11.9 \times 103 / \mu \mathrm{L}$ \\
\hline Platelets & 466 & 82 & 503 & $150-400 \times 103 / \mu \mathrm{L}$ \\
\hline Creatinin & 2,3 & 3,99 & 0,47 & $0,6-1,2 \mathrm{mg} / \mathrm{dL}$ \\
\hline $\mathrm{Na}^{+}$ & 145 & 142 & 139 & $136-145 \mathrm{mmol} / \mathrm{L}$ \\
\hline $\mathrm{K}^{+}$ & 4.0 & 3,9 & 4,2 & $3,5-5,1 \mathrm{mmol} / \mathrm{L}$ \\
\hline Total bilirrubin & 1,35 & 0,92 & 0,39 & $0,1-1,0 \mathrm{mg} / \mathrm{dL}$ \\
\hline Direct bilirrubin & 0,29 & & & $0-0,2 \mathrm{mg} / \mathrm{dL}$ \\
\hline C-Reactive protein (CRP) & 11,7 & 102 & 22,8 & $<3 \mathrm{mg} / \mathrm{L}$ \\
\hline INR & 1,2 & 3,5 & 1 & \\
\hline Activated partial thromboplastin time (aPTT) & 26,3 & 65,3 & 27,6 & $25-35 \mathrm{sec}$ \\
\hline \multicolumn{5}{|c|}{ Catecholamines } \\
\hline Serum metanephrine & & 9,30 & & $<0,46 \mathrm{nmol} / \mathrm{L}$ \\
\hline Serum normetanephrine & & 18,45 & & $<0,98 \mathrm{nmol} / \mathrm{L}$ \\
\hline Urinary metanephrine & & & 87,9 & $74-297 \mu \mathrm{g} / 24 \mathrm{~h}$ \\
\hline Urinary normetanephrine & & & 116,2 & $105-354 \mu \mathrm{g} / 24 \mathrm{~h}$ \\
\hline
\end{tabular}

Table 1: Analytical data (admission, before surgery and at ICU discharge).

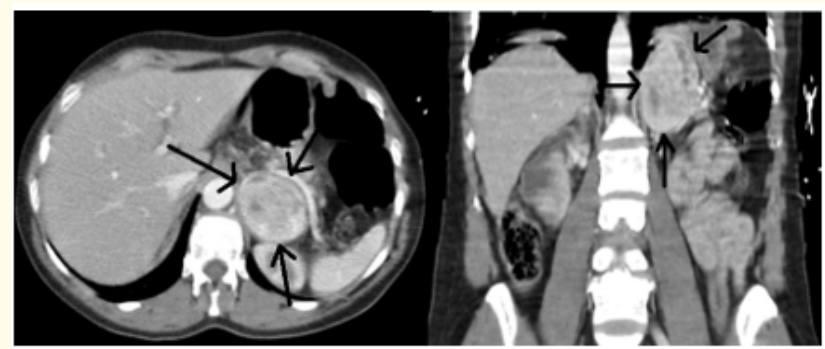

Figure 1: Axial and coronal abdominal CT scans showing an heterogenous nodular lesion in the left adrenal gland

(65 x 45mm) (arrows).
The patient was admitted in the Intensive Care Unit (ICU) with a shock with multiorgan disfunction (neurologic, respiratory, cardiovascular, and renal). The presence of a mass in the adrenal gland suggested the hypothesis of PMC.

In the ICU, she had a rapid deterioration of all organ disfunctions. The non-invasive ventilation trial failed, leading to sedation, intubation, and invasive mechanical ventilation. Renal replacement therapy (RRT) was initiated. She developed coagulopathy and fever. The cardiovascular component was the prominent feature, with sustained sinus tachycardia and increasing lactate, despite maintaining normal blood pressure without vasopressors. Initially, 
she had an exuberant extensive mottling in her limbs and abdomen. Soon some blue lesions appeared in her toes and fingers, these lesions rapidly coalesced to form extensive gloved black mummified lesions in both her inferior and superior limbs.

Two days after admission, she was submitted to an open left adrenalectomy. An intra-aortic balloon pump counter-pulse was implanted as preparation. She was initially managed with phentolamine (10mg intravenous) and, after clamping of the adrenal blood vessels, with noradrenaline. There were no significant haemodynamic fluctuations or other complications during surgery.

After surgery, she progressively recovered from multiorgan disfunction and was successfully weaned from ventilator and RRT (Figure 2).

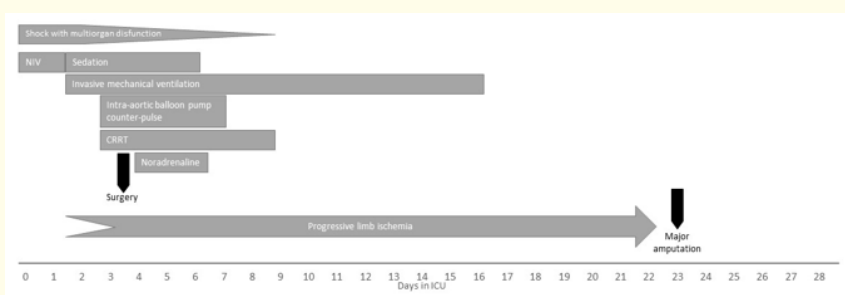

Figure 2: Chronogram of interventions in ICU.

Later, plasmatic metanephrines (Table 1) and histology of the surgical piece confirmed the diagnosis. It was a neoplasm composed of nests of epithelioid cells with abundant cytoplasm and evident nucleoli with areas of cellular pleomorphism, witch exhibited a diffuse expression of synaptophysin and chromogranin A in epithelioid cells and expression of Protein S100 in the sustentacular cells, with lack of expression of Melan A.

However, the lesions in her limbs persisted and evolved with an irreversible ischemia of both her feet and her right hand (Figure 3). Three weeks after admission, the patient went through a major amputation: left foot, right trans-tibiofibular and right hand.

After 28 days the patient was discharged from ICU to the nursery. She needed several interventions by plastic surgery followed by intense functional rehabilitation. She was discharged from hospital about 5 months after admission. After one year, she is fully adapted to her limb prosthetics and she regained her functional autonomy.

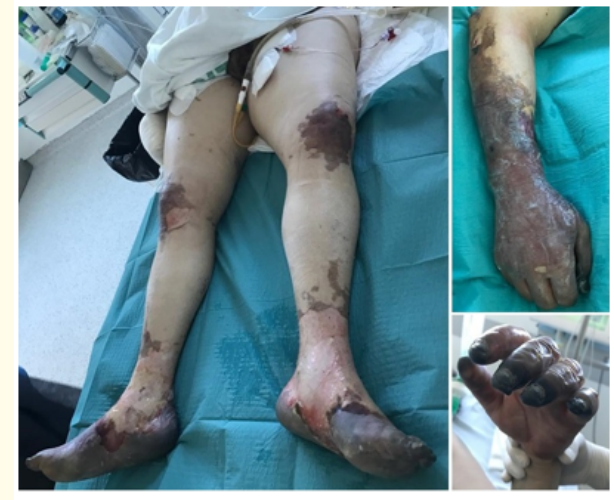

Figure 3: Macroscopic feature of the mummified limbs due to irreversible ischaemia.

\section{Discussion}

Pheochromocytomas are rare endocrine tumours with paroxysmal symptoms. This patient had an history of a recurrent headache that could be a pheochromocytoma-related symptom, as it is verified in about $30 \%$ of the cases [1].

Elevated plasma catecholamines result in a multitude of symptoms due to direct effect on cells and induced vasospasm $[2,4]$. This patient presented with abdominal pain probably due to mesenteric circulation compromise. Cerebral, renal and peripheric circulations impairment resulted in encephalopathy, renal dysfunction, and limb ischemia. Takotsubo cardiomyopathy was also prominent [2]. Acute inflammatory response is common in patients with pheochromocytoma [2], not necessarily meaning sepsis.

This patient met criteria for the diagnosis of PMC, regarding the rapid progression of multiorgan dysfunction in a patient with an adrenal mass, the presence of fever and the development of coagulopathy. The patient dramatically improved all dysfunctions after surgery, including complete reversal of the cardiomyopathy, supporting the diagnosis. The magnitude of metanephrines plasmatic levels (almost 20 times the upper limit) also validates the diagnosis and the histology of the surgical mass confirms the presence of a pheochromocytoma.

All pheochromocytomas should be resected surgical. Usually, 7 to 14 days of alfa-blockage is needed for adequate preoperative treatment [2]. However, PMC is an endocrinology emergency. ${ }^{3}$ In this case, emergency open surgery was the chosen approach, with- 
out time for standard preoperative management. Phentolamine is a short-acting drug, therefore an intra-aortic balloon pump counter-pulse was implanted to minimize haemodynamic compromise. This case provides further support that surgical resection is important for preventing mortality in patients with PMC. In fact, all European cases of PMC without surgical resection died despite conservative treatment. However, the appropriate time for surgery remains unclear [3].

Blue toe syndrome due to pheochromocytoma had previously been described and it is explained by small vessels vasospasm due to high levels of catecholamines [4,5]. In this case, the amount of plasmatic catecholamines resulted in extensive necrotic mummified lesions with irreversible ischemia of both her lower limbs and hands.

\section{Conclusion}

PMC is a rare presentation of an uncommon disease. The diagnosis of PMC is a clinical challenge, but the early recognition is of major importance. As illustrated by this report, a promptly surgery approach is a strategy to consider in these patients.

\section{Bibliography}

1. Falhammar H., et al. "Initial clinical presentation and spectrum of pheochromocytoma: a study of 94 cases from a single center". Endocrine Connections 7.1 (2018): 186-192.

2. Mitsis A., et al. "An unusual case of pheochromocytoma mimicking both acute coronary syndrome and central nervous system infection. Case report and literature review". Hellenic Journal of Cardiology 58.5 (2017): 372-377.

3. Kakoki K., et al. "Pheochromocytoma multisystem crisis treated with emergency surgery: a case report and literature review". BMC Research Notes 8 (2015): 758.

4. Mosquera Rey V., et al. "[Blue toe syndrome as a clinical finding of pheochromocytoma]". Medicina (B Aires) 78.5 (2018): 368-371.

5. Bandawar MS., et al. "An unusual cause of toe necrosis". Indian Journal of Endocrinology and Metabolism 17.1 (2013): 160162.

Volume 5 Issue 6 June 2021

(C) All rights are reserved by Elisabete Coelho., et al. 